Acute life-threatening episodes 254

Adult hemoglobin 203

Ambient air 27

Ammonia 38

Amylase 20

Antibiotics 51

Antioxidants 121

Apoptosis 91, 99, 221

ARDS 338

Astrocyte 99

Atelectasis 60

Betamethasone 273

Bilirubin 44,99

Birth asphyxia 27

Blood flow velocity 145

Brain hemodynamics and oxygenation 229

Bronchopulmonary dysplasia 82,181

Carbon monoxide 44

Carboxyhemoglobin 44

$\beta$-Casomorphin 160

Caspase-3 91

CCK receptors 20

Celecoxib 246

Cell-cell signaling 164

Chinese newborns 242

Chronic leptin administration 1

- lung disease 254

Clinical trials 338

Continuous positive airway pressure 254

C-reactive protein 105

Cyclooxygenase-2 246

Cytokine 152

Cytokines 66

Cytotoxicity 99

Desquamation 8

Development 20

Dexamethasone 113, 273

Dopamine receptors 229

Eicosanoids 66

Electron microscopy 197

Endotoxin 207

Environmental tobacco smoke 121

Erosion 197

Erythropoietin 15

Evaporimetry 197

Evolution 127

Extracellular matrix 113

Factor V Leiden 56

Fatty acids 66
Ferric reducing antioxidant power 121

Fetal hemoglobin 203

- origins 127

Fetus 38

Fibronectin 113

First trimester 187

Full-term newborns 187

Gastroesophageal reflux 254

Gene polymorphism 181

Gestational age 242

Glucose 197

Glutathione peroxidase 15

Grade I IVH 56

\section{Hemolysis 44}

Human 38

- milk 66

Hydrolyzed protein 160

Hypothermia 60

Hypothyroidism 273

Hypoxia-ischemia 207

Hypoxic-ischemic brain injury 15

IGF-I 91

Immunoglobulin G 221

Inflammation 207

Insulin 20

- resistance 127

Insulin-like growth factor-I 91

Interleukin 4181

Interleukin-1 82

Interleukin-10 207

Interleukin-6 105

Interstitial fluid, dermal 197

Intra-amniotic infection 152

Intraventricular hemorrhage 145

Kidneys 246

Laryngeal mask airway 217

Laser Doppler imaging 197

Leucine 236

Lipase 20

Lipid peroxidation 15,44

Logistic regression analysis 66

Lung development 113, 152, 164

- injury 221

Malondialdehyde 178

Matrix metalloproteinases 246

Meconium aspiration syndrome 51

Meconium-induced lung injury 73

Microdialysis 207 
Necrotizing enterocolitis 91

Neonatal adaptation 8

- , leptin 1

- lung disease 164

- - fluid 178

- mortality 27

- rat 15

Neurological assessment 187

Neutrophils 221

Newborn infant 121

- piglet 221

- resuscitation 27

Nitric oxide 73

Nuclear transcription factor kappa B 73

Nutrition 160

Opioid peptide 160

Oxidative stress 15

Oxygen 27

Partial liquid ventilation 60

Patent ductus arteriosus 145

Penile length 242

Perfluorocarbon 60

Perinatal brain injury 207

Photo-oxidation 44

Phototherapy 44

Piglet, leptin administration 1

Placenta 38

Polymerase chain reaction 181

Predictive adaptive response 127

Premature infant 44,160

- infants 236

Prematurity 82, 273

Preterm infants, sepsis 105

- neonates 56

Proliferating cell nuclear antigen 113
Protein carbonyls 44

- oxidation 44

Prothrombotic mutations 56

Pulmonary hypertension 221

- surfactant 73,140

Reactive oxygen species 44

Respiratory distress syndrome 140,152 , 217,283

- failure 73

Serum amyloid A in sepsis 105

Sheep 38

S-Nitrosohemoglobin 203

Soaps 8

SP-C analogs 338

Spectroscopy, near infrared 229

Stable microbubble test 140

Stable-isotope tracers 236

Steroids 273

Stratum corneum 8

Sudden infant death syndrome 254

Surfactant 8, 217

Thiobarbituric reactive substances 44

Thyroid 273

Thyroxine 273

Tidal volume 254

Total radical-trapping antioxidant parameter 121

Transdermal sampling 197

Trypsin 20

Uric acid 121

Vascular endothelial growth factor 246

Weaning 20 